\title{
Bioanalytical method development: considering information from a suite of complementary methods
}

"Successful method development will discover data contradictions, deal with dilemmas, resolve or report difficulties and issues, incorporate additional changes, respond to new requirements, and address incoherent expectations."

Keywords: assay synergy $\approx$ bioanalytical method development $\approx$ clinical diagnostics
" complementary methods $\approx$ immunoassay $\approx$ laboratory-developed tests $\approx$ LC-MS/MS

Bioanalytical method development can be viewed as a science, an art, a fundamental part of the high-stakes game of pharmaceutical development or an admixture of the above. Specifications can change during development; the latter stages of method development may inconveniently unearth new interferences; clinical samples may show unexpected variation; there may be conflicts with other timelines. Corporate stakes can be high, stratospheric even, before method development efforts have even begun. The completion of method development to the satisfaction of scientific, regulatory and commercial stakeholders can be difficult, even problematic.

A development scientist who develops a strong preference for a specific assay or instrument may, unfortunately, also develop a form of scientific myopia. Awareness of how other methods operate, their strengths and weaknesses, and what uses they have can appear to be information far afield from routine development concerns. During the development of a bioanalytical method or other high-complexity assays, however, a wider perspective may prove invaluable.

A preliminary bioanalytical method's performance may not meet all of management's metrics and requirements. When all requirements are held equal, the initial method's performance may significantly exceed some requirements, but this does not compensate for its shortfalls in other areas. This can create a difficult puzzle.

"This method has good resolution but needs to be much faster," has the faint scent of intrinsic conflict, "it must be cheaper, and have better precision, and specificity," and "by the way, we need it next week," has the sound of high expectations, high pressure and big trouble looming at the end of the next 7 days. Cost and turnaround time are notable real-world metrics, performance parameters that can work against analyte specificity and clinical sensitivity.

A preliminary immunoassay (IA) or LC-MS/MS method may possess performance meeting three out of four requirements. Still, the development scientist may arrive in desperate straits, unable to nail down all the needed details to the satisfaction of management and regulatory affairs. A rapid IA method may have cross-reactivity issues, while a high-specificity LC-MS/MS method may be a time- and operator-intensive high-complexity assay. Molecular genetic (MG) methods may require even more time and a different operator skill set, thus providing only a 'one or two out of four' solution, being neither timely nor detecting the desired analytes, rather, this method detects a genetic sequence or a specific mutation and is used to demonstrate a fundamental genetic basis for some conditions. Is it truly worth looking this far afield during development?

Reminiscing about ancient uses of IAs for blood typing, fluorescence methods for cytochrome quantitation, HPLC methods for small molecule quantitation, and large, bulky, esoteric mass spectrometers, who knew that these would eventually converge? That they would undergo different functional fusions and create more than one so-called hyphenated technique? We now have LC-MS/MS, RIA, RT-PCR, timeresolved immunofluorescence assays (TRIFA) and others. Such diverse methods, even the rudimentary non-hyphenated forms, may well be far afield from a development scientist's academic training. A stuck method development

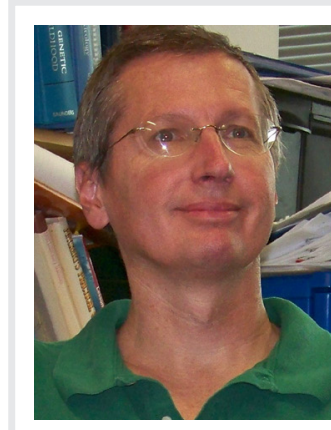

Mark E Fountain

2504 Greenland Lane, Austin, TX 78745, USA

Tel.: +| $5 \mid 2659$ |22|

E-mail: bootgoop@gmail.com 
scientist may find particular assistance from the different perspectives provided by an alternate method, insights potentially orthogonal to those already in the scientist's possession, data that the primary method cannot provide.

We all know that new method development can be easier if it is simply improving upon existing methods. Of course we all take care to remain aware of pre-existing pitfalls and limitations already reported in the literature that we read. Still, I admit my myopia here, my preference for looking in 'my literature.' The chemist looks in chemical literature and finds an LC-MS/MS method. The biologist's literature illustrates an IA method. The molecular biologist's literature illustrates a MG method solution to solve the same problem. A chemist asks, "Why look in biology or IA literature? Is there any reason to review MG research?” Yet, fundamentally, method development is not practiced in a vacuum. Rather, the development scientist walks in the footsteps of scientific predecessors and may stand on the shoulders of a scientist whose past work provides the underpinnings of the technology, knowledge and tools of today's laboratory.

As the number of requirements increase and the method complexity increases, the number of variables and the interactions also increase. With such complexity come new difficulties and complications arising through these interactions. What is a help, what is a hindrance? What tradeoffs can we afford? Which are issues to be resolved; which are correlations; what is due to causality? Approaches such as designed experiments and Taguchi methods, or TRIZ and systemic thinking can provide assistance; these pairs are for dealing with interacting variables and method robustness, or solving apparently unique problems and finding fundamental issues, respectively $[1,2,101]$. An additional issue arises as increasing complexity may also correspond to an increasing number of departments in the loop, both internal and external to method development teams.

A successful method needs to conform to clinical, regulatory and organizational requirements. Successful method development is not divorced from regulatory and commercial requirements. They are all a part of good science.

Meanwhile back in the laboratory, there is that fateful day during development when we get stuck. Not just a little stuck, but really stuck. The thought of a week in the library or a day discussing puzzling details with colleagues and collaborators may be just a faint whisper or a distant memory in the mind of a development scientist who is under the gun, in the pressurecooker with a severe case of tunnel vision and a critical deadline looming. Exorbitant expectations, dreaded deadlines, experimental deadends, the dreadful tone of the bells tolling doom, the bells seem to toll for thee. Sometimes, it is only at such a point that we, myself included, consider changing our approach. We old dogs who have passed our half-century mark keep hoping we're gaining a little insight. One who is quicker than me would have sooner recognized that repeating of the same laboratory behavior, and conducting experiments over and over again, while expecting a different result, is simply a sign of method development insanity. Luckily, most of us eventually hear a call-to-arms that beckons us to return to reality, to return to that which is realistically achievable.

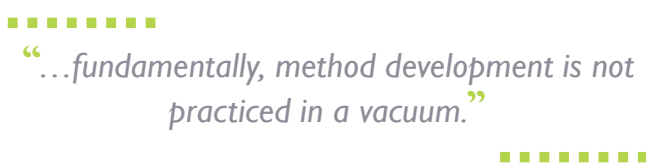

Ultimately, it probably does not matter if we are developing a quantitation, pre-clinical or other test method on a project for a US FDA submission, or a screening method or laboratory-developed test under CLIA guidelines. Issues and questions appear during development. Laboratory method improvements may solve the problem; a literature search may discover it to be a long-standing problem that simply requires documentation; or it may not even matter which approach one uses, so long as the problem is solved.

An experimentalist's first inclination during method development, myself included, is often more experiments. This approach was used by Thomas Edison and produced results - he was able to find more ways to not invent the electric light bulb. We can do more empirical observation, more of the usual modifications, more changing solvents and columns, polarity and $\mathrm{pH}$, time and temperature, tradeoffs of speed and specificity, separation and sensitivity; we can modify multiple parameters and we can tweak the method to improve performance. Unfortunately, whatever limitations a single method possesses can also limit the development scientist who is enamored of or married to that single method. Such an accidental ball and chain can result in a development scientist drowning in a sea of data, while trying to resolve apparent contradictions that cannot be resolved using that method. 
Today, TRIFA and LC-MS/MS methods are widely used, and MG techniques are becoming increasingly commonplace [3-5]. While such methods may not prove to be the final method used, in the cause of good science, information provided by such methods may give additional information that helps us to resolve nagging questions, those unyielding to the inquiry of the primary analytical method.

For example, while developing a second-tier LC-MS/MS steroid assay for abnormal IA samples, the preliminary LC-MS/MS method's sensitivity and specificity appeared adequate. There were, however, those troublesome samples that neither method resolved well, and borderline results that could represent potentially affected individuals or simply be false-positive results. Being a myopic chemist, literally and figuratively, my trip to the chemistry literature and looking up what other people had done to improve the assay yielded their quick fix doubling the sample size. Reviewing the literature that described the IA method, which this LC-MS/MS method was to complement, illuminated that adding a synthetic steroid to the extraction solvent effectively displaced the desired steroid from steroid-binding proteins and improved recovery. A separate trek through the IA literature also yielded surprises: a report of helpful pre-analytical variable corrections, insights into interferences and confounding factors, and even HPLC and GC-MS/MS data from over a decade ago that reported specific compounds that interfered with the IA [6-8]. A more recent GC-MS/MS paper described these so-called interfering compounds as specific urinary markers for the disorder [9].

Many current analytical methods can be traced back to wet methods, even steroid methods once used simpler qualitative and quantitative methods $[10,11]$. Fluorescence, IA and LC methods for some small molecules were developed decades ago. Today's scientist has current and historical information at their fingertips. The use of a discerning eye, to view both literature and experimental results, can discover and elucidate subtle details and notice differences that move development beyond where the older methods fall short. The new clues that emerge from empirical laboratory work during the method development process, which can sometimes help us unravel the next level of these highly complex puzzles. A successful new method provides an answer to a drug development, pre-clinical or clinical question.
If a method is in widespread use, there are probably multiple reasons. Newly published methods may illustrate that which is possible; widely used methods may demonstrate methods that are robust, feasible and state of the art in a regulated high-throughput environment. While methods and technology are continually evolving, we can only work with currently available technology. Alternate technologies are available for developing bioanalytical methods, and they may vary in practicality, robustness and how easily they are able to meet current regulatory requirements. Literature that describes methods in widespread use may clarify advantages and issues, as well as limitations, and can also contain information about that which makes other approaches impractical.

Even experimental work can sometimes help peel the blinders from our eyes. An IA method is robust and fast. An LC-MS/MS reanalysis of several hundred samples that the IA screen classified as normal was both humbling and educational. The samples, normal by IA, were also normal by LC-MS/MS analysis. Reanalysis of IA abnormal samples by LC-MS/MS, however, provided greater specificity and multianalyte detection that demonstrated obvious benefits and confirmed the value of LC-MS/ MS analysis. Such results forced me to concede a healthy respect for the adequacy of the IA method that resolved the normal and abnormal samples.

Additional literature forays for me, now in the medical literature, yielded other treasure and unexpected surprises: reports of similar steroid profiles produced by other transient conditions, interference from anti-coagulants, and that steroid therapies can produce false-positive or false-negative results [12-14].

To digress briefly, a parable: there once was a magical kingdom that was ruled by a wise and benevolent king who lived in a tall stone castle in the center of town. The king would look out of his window each day and see the townspeople gathered around the well that he had built with them several years ago. The people would draw their water, talk together and go about their daily business. Late one night, a wicked witch poisoned the well. The townspeople, all drinking from the well, went insane. Now the wise king, looking out his window, would see the townspeople drawing water, talking together and sadly lamenting the loss of their king's sanity, looking up to see their poor sad king at his window. 
The people continued to mourn their loss for several days. From the king's perspective, the poisoning was the problem; from the people's perspective, the problem was an insane king. The wise king considered the problem carefully: one world and two different perspectives. The king resolved to help his people, and took the quick way out, by going to the well the next morning, he took a drink of the water. The townspeople's celebrations went on for weeks; their king had regained his sanity.

All different perspectives produce different interpretations, a simple expedient that means we need not delve in the difficult question of objective sanity. Who knows? Maybe the wicked witch was a United Nations worker that dumped quinine into the well and cured malaria throughout the kingdom. Taken to the $\mathrm{N}^{\text {th }}$ degree, viewing different perspectives could imply that even scientists could be well advised to view issues from, hypothetically, a regulatory or commercial perspective. Admittedly, just the thought of this can make scientists shudder if they think about it for too long.

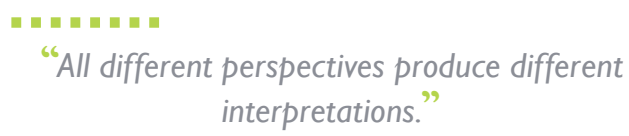
interpretations.'

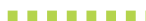

A completed bioanalytical method, its performance and results, will all be viewed from different perspectives once it has completed validation - looked at from scientific, regulatory and commercial perspectives - so shouldn't the development process also incorporate views from multiple perspectives? Seeing development issues from the perspective of a different assay methodology can indicate underlying pre-analytical and sample issues, causes of observed conflicts, alternative approaches and solutions, reasons for results, flawed literature pathways, and what other bioassay communities have experienced as practical, robust and possible.

Back in my literature forays, with more time spent wandering farther afield in related literature, this became a kind of a hobby for slow days, such forays became easier, even without comprehending all the material I was reading. A couple of papers that I found at this point, again quite by accident, rocked me back on my heels. The first paper, from the MG literature, provided corrections to published steroidogenesis pathways: species-specific differences [15]. These corrected pathways answered open questions surrounding some of the troublesome borderline results. The empirical results produced by the LC-MS/MS method suddenly made sense, and more data fit into a useful context. The second paper clarified that different mutations can be present, each diminishing steroid synthesis to a different extent, which provides a rationale for the experimentally observed continuum of steroid concentrations [16].

A method's performance is documented, in the end, by both validation and by being put into use. The developed method's strengths, weaknesses, good points and flaws, costs and limitations are all detailed during validation, but can be only an early indication of performance, with day to day use illuminating more changes to make, things missed or not thought of during development, and additional improvements needed. A method's validation can document the performance that a method is capable of and, ideally, this same performance is realistically, routinely, and consistently achieved. A method lacking the needed performance may simply be returned for required redevelopment.

Can we say that a library day and diverse literature can come to the rescue? Can talking with people in a different technical field who use a different assay methodology remove the tunnel vision of a development scientist? Can collaboration remove an experimentally impenetrable silo? Can looking elsewhere provide a dose of reality and help us move beyond the insights missing in instrumental data? In answer: maybe, possibly, could be, and it might be worth considering, respectively.

We must keep in mind the need for a rational review process, and not divorce method development from a careful and well-considered critique of a preliminary method's performance and the requirements it is slated to meet. Otherwise, starting requirements can contribute blinders or myopia, and trap the development team in a web of apparent contradictions. A continued ignorance of other approaches can hinder us, hiding alternative analytical solutions to issues that may be irresolvable by the primary analytical method. While the final method may be LC-MS/MS, evaluating IA and MG methods for the same purpose can inform development of what has been observed, and what is feasible using other technologies. A reality check might ask, "How do we integrate all that is needed into a coherent method?"

Successful method development will discover data contradictions, deal with dilemmas, resolve or report difficulties and issues, incorporate additional changes, respond to new requirements, and address incoherent expectations. Many sides of the multifaceted development dice must be 
examined and viewed from multiple angles and perspectives: platforms that permit the discovery of additional salient issues, the comprehension of incoherent contradictions created by scientists or discovered during development, as well as things that have escaped earlier scrutiny. Amidst a jumbled jungle we develop bioanalytical methods. With due diligence and an open mind, best practices and good science, we address complex problems and produce coherent solutions.

\section{Financial \& competing interests disclosure}

The author has no relevant affliations or financial involvement with any organization or entity with a financial interest in or financial conflict with the subject matter or materials discussed in the manuscript. This includes employment, consultancies, honoraria, stock ownership or options, expert testimony, grants or patents received or pending, or royalties.

No writing assistance was utilized in the production of this manuscript.

\section{References}

1 Schmidt SR, Launsby RG. Understanding Industrial Designed Experiments. Air Academy Press, Colorado Springs, CO, USA (1992).

2 Altshuller G. And suddenly the inventor appeared: TRIZ, the theory of inventive problem solving. Technical Innovation Center, Worcester, MA, USA (1994).

3 Maeda M, Hun Yang K, Tsuji A. Direct Solid-phase tine-resolved fluoroimmunoassay of $17 \alpha$-hydroxyprogresterone in dried blood spotted on filter paper. Analytical Sciences 7 , 699-702 (1991).

4 Schwarz E, Liu A, Randall $\mathrm{H}$ et al. Use of steroid profiling by UPLC-MS/MS as a second tier test in newborn screening for congenital adrenal hyperplasia: the Utah experience. Pediatr. Res. 66(2), 230-235 (2009).

5 Kosel S, Kösel S, Burggraf S et al. Rapid second-tier molecular genetic analysis for congenital adrenal hyperplasia attributable to steroid 21-hydroxylase deficiency. Clin. Chem. 51(2), 298-304 (2005).

6 Olgemoller B, Roscher AA, Liebl B, Fingerhut R. Screening for congenital adrenal hyperplasia: adjustment of 17-hydroxyprogesterone cut-off values to both age and birth weight markedly improves the predictive value. J. Clin. Endocrinol. Metab. 88(12), 5790-5794 (2003).

7 Fingerhut R. False positive rate in newborn screening for congenital adrenal hyperplasia $(\mathrm{CAH})$-ether extraction reveals two distinct reasons for elevated $17 \pm$-hydroxyprogesterone (17-OHP) values. Steroids 74(8), 662-665 (2009).

8 Wong T, Shackleton CH, Covey TR, Ellis G et al. Identification of the steroids in neonatal plasma that interfere with 17 alphahydroxyprogesterone radioimmunoassays. Clinical Chem. 38(9), 1830-1837 (1992).

9 Homma K, Hasegawa T, Takeshita E et al. Elevated urine pregnanetriol one definitively establishes the diagnosis of classical 21-hydroxylase deficiency in term and preterm neonates. J. Clin. Endocrinol. Metab. 89(12), 6087-6091 (2004).

10 Rudd BT, Cowper JM, Crawford N. The determination of plasma free hydrocortisone and corticocsterone by a combined fluorometric and modified porter-silber procedure. Clin. Chim. Acta 6, 686-695 (1961).

11 Cox RI. A method for the quantitative determination in urinary extracts of c21 17:20-dihydroxy-20-methylsteroids. Biochem. J. 52(2), 339-344 (1952).
12 Cavarzere P, Samara-Boustani D, Flechtner I et al. Transient hyper-17-hydroxyprogesterone mia: a clinical subgroup of patients diagnosed at neonatal screening for congenital adrenal hyperplasia. Eur. J. Endocrinol. 161(2), 285-292 (2009).

13 Fingerhut R, Dame T, Olgemoller B. Determination of EDTA in dried blood samples by tandem mass sepctrometry avoids serious errors in newborn screening. Eur. J. Pediatr. 168, 553-558 (2009).

14 White PC. Neonatal screening for congenital adrenal hyperplasia. Nat. Rev. Endocrinol. 5(9), 490-498 (2009).

15 Bird IM, Conley AJ. Steroid biosynthesis: enzymology, integration and control. In: Genetics of Steroid Bioosynthesis and Function. Mason JI (Ed.). Taylor and Francis, London, UK 1-35 (2002).

16 White PC, Speiser PW. Congenital adrenal hyperplasia due to 21-hydroxylase deficiency. Endocr. Rev. 21(3), 245-291 (2000).

\section{Website}

101 Bartlett G. Systemic thinking. www.systemicthinking.com (Accessed 14 November 2011) 